\title{
Docosahexaenoic acid alleviates cartilage degradation by mediating apoptosis and autophagy via JNK and p38 signaling pathways in human chondrocytes and osteoarthritis rat model
}

\section{Zishun Gong}

Capital Medical University Affiliated Beijing Friendship Hospital

Haomiao Yu

Capital Medical University Affiliated Beijing Friendship Hospital

Guodong Wang

Beijing Tongren Hospital

\section{Ruiqi Cao}

Capital Medical University Affiliated Beijing Friendship Hospital Heyong Yin

Capital Medical University Affiliated Beijing Friendship Hospital

\section{Lifeng Ma}

Capital Medical University Affiliated Beijing Friendship Hospital

Ai Guo ( $\nabla$ guoaij@139.com )

Capital Medical University Affiliated Beijing Friendship Hospital https://orcid.org/0000-0002-40425682

\section{Research Article}

Keywords: Osteoarthritis, Docosahexaenoic acid, Apoptosis, Autophagy, Chondrocyte.

Posted Date: February 23rd, 2022

DOl: https://doi.org/10.21203/rs.3.rs-1362485/v1

License: (c) (1) This work is licensed under a Creative Commons Attribution 4.0 International License. Read Full License 


\section{Abstract}

Background: Osteoarthritis $(\mathrm{OA})$ is a degenerative joint disease which occurs commonly in the elderly. Docosahexaenoic acid (DHA) is considered to play an anti-inflammatory role in the inflammation process of the human body. The aim of this study is to observe the effect of DHA on the proliferation, apoptosis, autophagy, and related signaling pathway in human OA chondrocytes and the effect of DHA on cartilage degeneration in vivo.

Methods and Results: Human OA chondrocytes model was established by IL-1 $\beta$ while OA rat models were prepared by anterior cruciate ligament transection and medial meniscectomy. The results showed that DHA promoted chondrocyte proliferation and decreased the percentage of apoptotic chondrocytes.

Transmission electron microscopy (TEM) analysis showed more autophagic bodies in the cytoplasm with the treatment of DHA. Cartilage samples from the OA + DHA group showed thicker cartilage, decreased degradation and increased collagen II positive cell rate compared to the OA group, while the Mankin score was significantly lower. Additionally, DHA resulted in reduced mTOR expression and LC3-I/II ratio, increased Beclin-1 and Bcl-2 expression measured by Western-blot analysis. The mRNA expression of JNK and p38 was reduced compared to the IL-1 group. However, after intervention with 3-Methyladenine (3-MA, an autophapy inhibitor), the effect of DHA was blocked.

Conclusions: DHA promoted chondrocyte proliferation, decreased cell apoptosis, and increased autophagic activity in OA chondrocytes. DHA could reduce cartilage degradation and increase collagen II secretion in vivo. We hypothesized that the underlying mechanism involved the suppression of the JNK and p38 signaling pathways.

\section{Introduction}

Osteoarthritis (OA) is a degenerative joint disease, which usually affects the elderly. Studies have shown that more than half of the people over 65 years of age have symptomatic or radiological OA[1]. With an increase in the aging population around the world, there has been a gradual increase in the prevalence of $\mathrm{OA}[2]$, causing a huge social and economic burden. Numerous molecular pathways and biomarkers are involved in the pathological process of OA, such as MMPs, TIMPs, nitric oxide, sodium nitroprusside, p53, c-myc, mitogen-activated protein kinase/c-JunN terminal kinase (MAPK/JNK), PARP-1, Toll-like receptors $1 / 2($ TLR1/2), tumor necrosis factor- a (TNF-a), etc. $[3,4]$.

Apoptosis also called programmed cell death, plays a critical role in maintaining the homeostasis of various tissues in the adult human body. Dysregulation of apoptosis leads to pathological states, such as cancer, developmental anomalies, and degenerative diseases[5]. Autophagy is a metabolic process in which the lysosomes degrade damaged proteins and organelles. It is an important survival mechanism when cells are under stress, and it plays an important role in hypoxia, starvation, and other stress conditions[6, 7]. 
Docosahexaenoic acid (DHA) is an n-3 polyunsaturated fatty acid, which is commonly found in deep-sea fish oil and vegetable oil, making it an important nutrient in the diet[8]. According to recent studies, DHA has extensive and definite anti-inflammatory effects on multiple systems[9], but its activity on the inflammatory response of OA chondrocytes and cartilage is still unclear. We speculated that DHA played an anti-inflammatory role in OA by regulating apoptosis and autophagy of chondrocytes. Thus, we determined the effect of DHA on OA chondrocytes in cell culture and in an OA rat model.

\section{Material And Methods}

\section{Isolation, culture, and identification of chondrocytes}

The surface of cartilage was resected from the normal area of the femoral head in patients with femoral neck fracture undergoing artificial femoral head replacement. The cartilage was from seven patients (2 males and 5 females with no prior relevant surgical history, aged 63-82 years). Ethics approval was granted by the Bioethics Committee of Beijing Friendship Hospital, Capital Medical University, China (approval code 2020-P2-205-01). Informed consent was obtained from all patients before surgery. The cartilage tissue was rinsed in PBS (Solarbio, Wuhan, China) and cut into small pieces. Next, $0.2 \%$ type II collagenase (Sigma, MO, US) was added to isolate the chondrocytes, and the vessel was vibrated in a $37^{\circ} \mathrm{C}$ water bath for $3-4 \mathrm{~h}$. The supernatant was centrifuged at $1200 \mathrm{rpm}$ for $5 \mathrm{~min}$, and the precipitated cells were rinsed with PBS, incubated with DMEM medium (Solarbio, Wuhan, China) supplemented with $10 \%$ fetal bovine serum (Sigma, MO, US), $100 \mathrm{IU} / \mathrm{mL}$ penicillin and $100 \mu \mathrm{g} / \mathrm{mL}$ streptomycin, at $37^{\circ} \mathrm{C}$ in humidified air containing $5 \% \mathrm{CO}_{2}$.

We used Safranin 0 and toluidine blue staining to identify chondrocytes. Cells were seeded in cell climbing sheets, dehydrated with $95 \%$ ethanol, stained with safranine 0 and toluidine blue, respectively, and then cleaned and sealed with neutral resin.

In the first passage, chondrocytes were cultured up to $80 \%$ confluency, counted, and divided into four groups. The control group was treated with normal saline in the same amount as other groups. The IL-1 group was stimulated with $100 \mathrm{pg} / \mathrm{mL}$ IL-1 (Sigma, MO, US) to establish the OA chondrocyte model. The IL-1 + DHA group was pre-treated with $50 \mu \mathrm{g} / \mathrm{mL}$ DHA (Sigma, MO, US) $1 \mathrm{~h}$ before $100 \mathrm{pg} / \mathrm{mL}$ IL-1. The IL$1+$ DHA + 3-MA group was pre-treated with $50 \mu \mathrm{g} / \mathrm{mL} \mathrm{DHA}$ and $5 \mathrm{mmol} / \mathrm{L} 3-\mathrm{MA}$ (Sigma, MO, US), an autophagy inhibitor $1 \mathrm{~h}$ before treatment with $100 \mathrm{pg} / \mathrm{mL} \mathrm{IL}-1$. Protein and mRNA expression was measured $24 \mathrm{~h}$ after stimulation.

\section{Animal Models}

A total of 24 Sprague-Dawley male rats (8 weeks old) were divided into the control group, OA group, Sham + DHA group, and OA + DHA group ( $n=6$ each). The control group and OA group were fed a normal AIN-93G diet, and the Sham + DHA group and OA + DHA group were fed an AIN-93G diet rich in DHA (add $5 \mathrm{~g}$ DHA per $\mathrm{kg}$ diet). In the OA group and OA + DHA group, OA rat models were prepared by anterior cruciate ligament transection and medial meniscectomy. The Sham + DHA group was compared by sham 
operation (only the articular capsule was cut open). All rats were euthanized by cervical dislocation after 6 weeks, while the cartilage and subchondral bone of the knee on the femur and tibia were removed and stored in formaldehyde solution for further histology. All procedures were approved by the Bioethics Committee of Beijing Friendship Hospital, Capital Medical University, China (approval code 2020-P2-20501).

\section{Cell viability analysis}

The cell viability analysis was done using the CCK8 (cell counting kit-8) assay. Chondrocytes were seeded in 96 -well plates $\left(5,000\right.$ cells/well) and incubated at $37^{\circ} \mathrm{C}$ for $24 \mathrm{~h}$. Then the cells were divided into four groups and pre-treated as previously mentioned. After $24 \mathrm{~h}, 10 \mu \mathrm{L}$ of CCK-8 (Solarbio, Wuhan, China) reagent was added to each well, and the cells were incubated for another $4 \mathrm{~h}$. The absorbance was measured using a multi-functional microplate reader (Molecular Devices, Sunnyvale, CA, USA) at $490 \mathrm{~nm}$. The relative cell viability (RCV) to the controls was calculated using the following equation:

$\mathrm{RCV}=\left(\mathrm{OD}_{\text {test }}-\mathrm{OD}_{\text {blank }}\right) /\left(\mathrm{OD}_{\text {control }}-\mathrm{OD}_{\text {blank }}\right) \times 100 \%$.

\section{Flow Cytometry Assay}

Chondrocytes were cultured with various treatments for $24 \mathrm{~h}$ in 6-well plates, and the apoptosis rates were determined by Annexin V-FITC/PI apoptosis kit according to the instructions using flow cytometry (FCM) analysis. The cells were resuspended in $1 \mathrm{X}$ binding buffer and stained with $5 \mu \mathrm{L}$ of Annexin V-FITC and $5 \mu \mathrm{L}$ of propidium iodide $(\mathrm{PI})$, and then cultured in the dark at $25^{\circ} \mathrm{C}$ for 15 minutes. After culture, the samples were detected by flow cytometry. The cells considered to be in early apoptosis were Annexin VFITC positive and PI negative; Annexin V-FITC and PI were both positive in late apoptotic or dead cells. The experiment was performed thrice.

\section{Western blot analysis}

The cells were washed in ice-cold PBS and lysed in total protein lysis buffer (Ukzybiotech, Beijing, China) supplemented with $1 \mathrm{mg} / \mathrm{mL}$ protease inhibitor cocktail (Roche, US) at $4^{\circ} \mathrm{C}$ for $30 \mathrm{~min}$. After protein extraction, the BCA (Solarbio, Wuhan, China) method was used for protein quantification. Next, SDS-PAGE electrophoresis was performed at $95^{\circ} \mathrm{C}$ for $5 \mathrm{~min}$, with the final sample concentration of $0.8 \mu \mathrm{g} / \mu \mathrm{L}$ adjusted by RIPA buffer. After electrophoresis, the protein bands were transferred to a 0.45 um PVDF membrane (Millipore, US) for $90 \mathrm{~min}$. The membrane was completely immersed in 5\% BSA-TBST and incubated in a horizontal shaker for $1 \mathrm{~h}$. Then the membrane was incubated with 5\% BSA-TBST and diluted primary antibody to mTOR (1:1000) (Cell signaling, MA, US), Beclin-1 (1:2000) (Abcam, CA, UK), Bcl-2 (1:1000) (Abcam, CA, UK), light chain 3 (1:2000) (Abcam, CA, UK), and $\beta$-actin (1:1000) (ZS, Beijing, China) overnight at $4^{\circ} \mathrm{C}$. The blots were then developed using a horseradish peroxidase (HRP)-conjugated goat anti-rabbit or goat anti-mouse IgG secondary antibody (1:10000) (Jackson, US), incubated at room temperature for $40 \mathrm{~min}$, and subjected to signal development using enhanced chemiluminescence (ECL) substrate (Thermo, US). $\beta$-actin was used as an internal parameter; the expression of each protein was 
equal to the gray value of target protein/ $\beta$-actin. Each sample was analyzed thrice, and the mean values of the immunoblot band were calculated.

\section{Reverse transcription-quantitative polymerase chain reaction (RT-qPCR)}

The RNA was extracted using the Trizol (Invitrogen, US) reagent. The extracted RNA was reverse transcribed into single-stranded cDNA. Quantitative RT-PCR reactions were performed in triplicate using a 7500 Real-Time PCR System (ABI, US) with SYBR Green qPCR Master Mix (ABI, US). The expression of target genes relative to $\beta$-actin was calculated using the $2^{-\triangle \Delta C T}$ method. The primers were designed as follows:

\begin{tabular}{|c|c|c|c|}
\hline Primer & Sequences ( $5^{\prime}$ to $\left.3^{\prime}\right)$ & Number of bases & Lengths of products \\
\hline \multirow[t]{2}{*}{$\beta$-Actin } & F: TCCATCATGAAGTGTGACGT & 20 & \multirow[t]{2}{*}{ 154bp } \\
\hline & R: GAGCAATGATCTTGATCTTCAT & 22 & \\
\hline \multirow[t]{2}{*}{ JNK } & F: TTCACATTGAGCAGAGCAGGCATA & 24 & \multirow[t]{2}{*}{$132 b p$} \\
\hline & R: ACTTGTCAGGAGCAGCACCATTC & 23 & \\
\hline \multirow[t]{2}{*}{ ERK } & F: ACCGTGACCTCAAGCCTTCCA & 21 & \multirow[t]{2}{*}{$216 b p$} \\
\hline & R: CCAGAATGCAGCCTACAGACCAA & 23 & \\
\hline \multirow[t]{2}{*}{ PERK } & F: CCTCGCAGTGGCAATGAGAAGT & 22 & \multirow[t]{2}{*}{$205 b p$} \\
\hline & R: TCCAGTCAGCAACCGAAACCTTT & 23 & \\
\hline \multirow[t]{2}{*}{ IKBA } & F: AGACGAGGAGTACGAGCAGATGG & 23 & \multirow[t]{2}{*}{$182 b p$} \\
\hline & R: CCTTCACCTGGCGGATCACTTC & 22 & \\
\hline \multirow[t]{2}{*}{ PI3K } & F: GGTTGGTGGCTGTTCTTACTGTC & 23 & \multirow[t]{2}{*}{$200 \mathrm{bp}$} \\
\hline & R: CAAGTCTGGCTGGAATGATGCTAT & 24 & \\
\hline \multirow[t]{2}{*}{ GRP78 } & F: CCGAGGAGGAGGACAAGAAGGA & 22 & \multirow[t]{2}{*}{$160 \mathrm{bp}$} \\
\hline & R: TCAGGAGTGAAGGCGACATAGGA & 23 & \\
\hline \multirow[t]{2}{*}{ P38 } & F: CCGAGCGTTACCAGAACCTGTC & 22 & \multirow[t]{2}{*}{ 107bp } \\
\hline & R: GGAGAGCTTCTTCACTGCCACAC & 23 & \\
\hline \multirow[t]{2}{*}{ Bax } & F: ATTGCCGCCGTGGACACAGA & 20 & \multirow[t]{2}{*}{$138 b p$} \\
\hline & R: CAGGGCCTTGAGCACCAGTTTG & 22 & \\
\hline
\end{tabular}

\section{Transmission electron microscopy}


Transmission electron microscopy was used to observe chondrocyte degeneration and autophagosomes. Chondrocytes were collected, fixed with 1\% OsO4 in PBS (pH 7.0) for $2 \mathrm{~h}$, and washed thrice in PBS. Then the cells were dehydrated by serial washing with ethanol. The samples were subsequently incubated in a mixture of alcohol and isoamyl acetate and embedded in gold-palladium. Ultra-thin sections were produced and observed under a transmission electron microscope (TEM, Hitachi, Japan).

\section{Cartilage and subchondral bone histology}

The distal femur and proximal tibia fixed with formalin were decalcified in 0.5 M EDTA for 6 weeks and then embedded in paraffin. A series of $4 \mu \mathrm{m}$ sections were made on the sagittal plane along the middle of the medial femoral condyle of the knee joint. This area was the weight-bearing surface of the medial femoral condyle, showing the earliest and most serious histological abnormalities in the joint [10]. Next, sections were stained with $\mathrm{H} \& \mathrm{E}$, toluidine blue, and safranin-O Fast Green to evaluate histological changes. A modified version of Mankin's grading score system was used on safranin-O Fast Green staining to assess cartilage damage [11]. Images with an original magnification of $400 \times$ were obtained by optical microscope. Each image was evaluated thrice by three experienced observers, and the average score was used in statistical analyses.

\section{Immunohistochemical analysis}

Next, $4 \mu \mathrm{m}$ formalin-fixed paraffin sections were made for each sample and then deparaffinized in xylene, hydrated using graded ethanol, rinsed in PBS for 5 minutes, and incubated in $0.125 \%$ trypsin (Sigma) for 15 minutes in $37^{\circ} \mathrm{C}$. The sections were incubated in $0.3 \% \mathrm{H}_{2} \mathrm{O}_{2}$ /methanol for 30 minutes to quench endogenous peroxidase activity and were rinsed thrice with PBS for 3 minutes. Then the slides were blocked with $100 \mu \mathrm{L}$ normal goat serum (Solarbio, Beijing, China) for 15 minutes at room temperature. After blocking, the slides were incubated overnight at $4^{\circ} \mathrm{C}$ with rabbit anti-Collagen-II antibody (1:200, Bioss, Beijing, China). Secondary biotinylated goat anti-rabbit antibody (Bioss, Beijing, China) was added for 30 minutes on the second day, followed by incubation with avidin-biotin complex reagent (Bioss, Beijing, China) containing horseradish peroxidase for $30 \mathrm{~min}$. The sections were then stained with 3,3'diaminobenzidine (DAB) (Sigma). The positive rate was calculated by positive cells/total cells.

\section{Statistical analysis}

The data are expressed as the mean and standard deviation (sd). Statistical analysis was performed using SPSS 19.0. The student's t-test was used to compare two groups. One-way analysis of variance was used for multiple comparisons between independent groups. A statistically significant difference was considered as $P<0.05$.

\section{Results}

\section{DHA increased human chondrocyte viability}


There was no significant difference between the RCV of the IL-1 group and the control group $(P=0.3)$. However, after the addition of DHA, the cell proliferation rate increased by $6.53 \%$ compared to the control group ( $P=0.017)$. After the addition of 3-MA into the medium, the change of RCV was not significant compared to the IL-1 + DHA group $(P=0.651)$. These results indicated that DHA acted as a positive regulator for the cell proliferation of osteoarthritic chondrocytes (Fig. 1f).

\section{DHA suppressed IL-1 Induced Chondrocyte Apoptosis}

Flow cytometry was used to evaluate cell apoptosis to further explore the effects of DHA on the apoptosis of human chondrocytes. The results showed that when IL-1 $\beta$ acted on chondrocytes, there was a significant increase in the percentage of apoptotic chondrocytes $(P<0.001)$. After the treatment with DHA, the percentage of apoptotic chondrocytes was decreased $(P<0.001)$. However, when IL-1 induced chondrocytes were incubated with DHA and 3-MA, the percentage of cell apoptosis increased significantly compared to the IL-1 + DHA group $(P<0.001)$ (Fig. 1e).

\section{DHA elevated Autophagic activity of OA chondrocytes}

Cells were divided into four groups (control, IL-1 group, IL-1 + DHA group, IL-1 + DHA + 3MA group), and the expression of mTOR, Beclin-1, Bcl-2, and LC3-I/II were detected. The results showed that mTOR expression $(P=0.011)$ and LC3-I/II ratio $(P<0.001)$ was elevated in OA chondrocytes, while the expression of Beclin-1 $(P=0.001)$ and $\mathrm{Bcl}-2(P=0.001)$ was significantly decreased. Compared to the IL-1 group, DHA decreased mTOR expression $(P<0.001)$ and LC3-I/II $(P<0.001)$ and increased the expression of Beclin-1 $(P<0.001)$ and Bcl-2 $(P=0.003)$. However, when the cells were incubated with DHA and autophagy inhibitor 3-MA, mTOR expression and LC3-I/II increased significantly (both $P<0.001$ ), and the expression of Beclin-1 $(P<0.001)$ and Bcl-2 $(P=0.001)$ decreased, indicating that the effect of DHA was blocked by 3-MA (Fig. 2a, 2b).

Chondrocytes were observed via TEM to detect autophagic bodies and other organelles. In the control group, chondrocytes appeared with a normal structure, with several autophagic bodies with bilayer membrane structure in the cytoplasm. In the IL-1 group, fewer autophagic bodies were observed in chondrocytes. In the IL-1 + DHA group, a greater number of autophagic bodies and rough endoplasmic reticulum were found in the cytoplasm. In the IL-1 + DHA + 3-MA group, fewer autophagic bodies and more vacuoles were seen in the cells. (Fig. 2c)

\section{DHA reduced JNK and p38 signaling pathway expression in OA chondrocytes}

The mRNA expression of eight signaling pathways (JNK, ERK, PERK, IKBA, GRP78, PI3K, p38, and Bax) was detected by RT-PCR. The results implied that compared to the control group, the mRNA expression of JNK, ERK, GRP78, PI3K, and p38 in the IL-1 group was elevated (all $P<0.05)$. After the addition of DHA, the expression of JNK and p38 reduced compared to that in the IL-1 group ( $P=0.002,0.016$ respectively). 
Furthermore, when cells were cultured with DHA and 3-MA, JNK and p38 expression increased significantly (both $P<0.001$ ) (Fig. 3).

\section{DHA alleviated cartilage damage and reduced the Mankin score in the OA rat model}

Cartilage damage in the knees was shown by H\&E, toluidine blue, and safranin-O Fast Green staining (Fig. 4a) and was evaluated using a modified histopathological Mankin grading score (Fig. 4b). Sections from the OA group showed more erosion, less thickness of the cartilage, and more irregular cartilage surface than the control group and the Sham + DHA group. Total Mankin scores of the OA group were significantly higher than the control group and the Sham + DHA group (both $P<0.001$ ). In the OA + DHA group, Mankin scores were also higher than that of the control group and the Sham + DHA group (both $P$ $<0.001)$. However, when compared with the OA group, cartilage samples from the OA + DHA group showed thicker cartilage and decreased degradation. Mankin scores were significantly lower in the OA + DHA group compared to the OA group (6.67 \pm 1.79 vs. $7.93 \pm 1.55$, respectively; $P=0.029)$.

\section{DHA increased collagen II positive chondrocyte rate in OA rat model}

Collagen II expression of the cartilage was measured by immunohistochemical staining (Fig. 5). The sections showed significantly fewer positive cells in the OA group compared with the control and the Sham + DHA group (both $P<0.001$ ). However, when the OA model rats were fed DHA, there was a significant increase in the collagen II positive cell rate $(42.56 \pm 5.61 \%$ vs. $57.94 \pm 6.24 \%$, respectively; $P<$ 0.001), indicating more collagen II expressions in the cartilage.

\section{Discussion}

Osteoarthritis, also known as degenerative arthropathy, is characterized by articular cartilage degeneration, subchondral bone remodeling, synovitis, and osteophytes formation [12, 13]. OA leads to joint pain, stiffness, deformity, and dysfunction. It occurs commonly in the elderly and is associated with limb disability and a severe decline in the quality of life of the patients $[14,15]$. According to statistics, the incidence of osteoarthritis accounts for $15-18 \%$ of the total population and includes approximately $80 \%$ of elderly people over 75 years old [16]. The major treatment of OA includes reducing joint bearing, medicine, physical therapy, and surgery, which are mainly symptomatic treatment rather than etiological treatment [17].

DHA (docosahexaenoic acid) is a type of n-3 polyunsaturated fatty acid (n-3 PUFA)[18]. Since it is not synthesized by the human body and needs to be ingested from food, DHA along with EPA are called "essential fatty acids" Recent studies have suggested that DHA and EPA play a strong anti-inflammatory role in the inflammation process of the human body[19-21]. In LPS-induced THP-1 cells, $100 \mu \mathrm{mol} / \mathrm{L}$ DHA could reduce the level of TNF-a, IL-1 $\beta, I L-6$, and other inflammatory factors [22]. When rats were fed 
with fish oil containing DHA and EPA, ICAM-1 expression on the surface of macrophages was decreased, and the synthesis of TNF-a, IL-1 $\beta$, and IL- 6 was significantly reduced [23]. Wang et al. [24] found that DHA inhibited the overexpression of IL-1 and MMP-13 through the p38-mediated MAPK pathway, thereby alleviating articular cartilage degeneration. In the study by Mehler [25], daily supplementation of a dogs' diet with EPA and DHA could shift the blood fatty acid concentrations correlated to the relief of clinical signs associated with OA in dogs. Xu's study [26] showed that DHA had anti-apoptotic effects in IL-1 $\beta$ induced human chondrosarcoma cell death through the MAPK signaling pathway. Gruenwald $\mathrm{J}$ et al. [27] demonstrated that patients with 26 weeks of oral administration of glucosamine sulfate and omega-3 polyunsaturated fatty acids (EPA and DHA) had a lower WOMAC pain score than patients with glucosamine sulfate only. These studies suggest that DHA had anti-inflammatory effects on the body. However, the effect of DHA on the apoptosis and autophagy of articular chondrocytes has not been studied, and the mechanism is still unclear.

Apoptosis and Autophagy are both important pathophysiological processes during cartilage degeneration. Apoptosis is a form of programmed cell death in which a predetermined sequence of events leads to the removal of old, unnecessary, and unhealthy cells without releasing harmful substances to the surrounding area[28]. Apoptotic cell death is usually detected in osteoarthritis (OA) cartilage. This is related to matrix degradation and calcification, suggesting that cell death /survival plays a role in the pathogenesis of $O A[29,30]$. IL-1 $\beta$ is a common proinflammatory cytokine that induces chondrocyte apoptosis in vivo and in vitro, resulting in cartilage matrix degradation and joint inflammation, thereby contributing to OA progression[31, 32]. Our study showed that IL-1 $\beta$ significantly increased chondrocyte apoptosis, which was consistent with the results of previous studies[33]. However, treatment with DHA reduced the percentage of apoptotic chondrocytes induced by IL-1 $\beta$. Furthermore, chondrocyte viability increased in the presence of DHA. These results suggested that DHA exhibited a chondroprotective effect on OA chondrocytes by inhibiting the cell apoptosis process.

Autophagy is a cellular self-protection mechanism, which keeps energy regeneration and nutrition recycling $[34,35]$. In the case of inflammation, exogenous chemicals, oxides, infection, and hypoxia, autophagy maintains cellular homeostasis by removing damaged organelles and intracellular unfolded proteins, playing an important role in cell survival under stress conditions [36, 37]. It is known that mTOR, Beclin-1, and LC3 play important roles in all autophagy-related proteins; mTOR holds a vital negative control on the cell autophagy associated with the participation of LC3-I and LC3-II[38]. Beclin-1 is involved in the initial stage of autophagolysosomal formation[39]. The process of autophagy requires the participation of LC3, which has two forms: LC3-I and LC3-II. During autophagy, LC3-I is transformed to LC3-II, and then LC3-II binds to autophagic vesicles[40]. Cheng et al.[41] found that autophagy affected chondrocyte degeneration. In OA models in vitro and in vivo, autophagy inhibitor 3-MA inhibited autophagy, aggravating chondrocyte damage and knee cartilage degeneration. In a rat OA model, intraarticular injection of Torin (a specific inhibitor of mTOR pathway) upregulated autophagy, decreased MMP13 and VEGF expressions [42], enhanced TIMP-1, and Beclin-1 expression, and LC3-II/I ratio, and alleviated the degeneration of articular cartilage[43]. 
In our study, an in vitro OA model was established to investigate the function of DHA on cell autophagy. The result showed that mTOR expression and LC3-I/II ratio were elevated in OA chondrocytes, while the expression of Beclin-1 and Bcl-2 was significantly decreased, suggesting that IL-1 inhibited autophagy. Representative TEM images also found fewer autophagic bodies in the cytoplasm. After the addition of DHA, mTOR expression and LC3-I/II ratio were significantly lower than that of the IL-1 group, while the protein synthesis of Beclin-1 increased significantly. This process demonstrated that DHA could activate autophagy in inflammatory chondrocytes. Similarly, Wann AK [44] found both EPA and DHA at 0.1 and 1 $\mu \mathrm{M}$ concentrations, respectively, could significantly reduce IL-1 $\beta$-mediated cartilage degradation through competitive inhibition of the arachidonic acid oxidation pathway. However, after the autophagic pathway was inhibited by 3-MA, mTOR expression and LC3-I/II ratio increased again while Beclin-1 decreased, indicating that the activation of autophagy by DHA was blocked by 3-MA. TEM images showed that more autophagic bodies and rough endoplasmic reticulum were generated by the inflammatory chondrocytes when DHA existed. On the contrary, while 3-MA was added into the medium, fewer autophagic bodies numbers and more vacuoles were observed.

The chondroprotective effect of DHA was also demonstrated in OA rat models. The models were established by anterior cruciate ligament transection and medial meniscectomy. The results of histological staining showed more erosion, less thickness of the cartilage, higher Mankin scores, and fewer collagen II expressions. However, when OA model rats were fed DHA, histology sections showed less erosion, Mankin scores decreased, and collagen II expressions were enhanced. These results suggested that DHA could alleviate the damage and degeneration of OA articular cartilage.

The potential pathway of DHA action on autophagy was detected by RT-PCR. The expression of eight signaling pathways (JNK, ERK, PERK, IKBA, GRP78, PI3K, P38, Bax) was measured, and the results showed that IL-1 increased mRNA expression of five pathways: JNK, ERK, PI3K, p38, and Bax. However, after treatment with DHA, the expression of JNK and p38 plummeted significantly, indicating that JNK and p38 played a significant role in this procedure. When 3-MA was added into the medium, JNK and p38 expression increased significantly, suggesting that the inhibitory effect of DHA on the JNK and p38 signaling pathway was blocked by 3-MA, and consequently, the autophagic activity. This result implied that DHA activated autophagy by inhibiting the expression of JNK and p38 signaling pathways. In other studies, Phitak T et al.[45] found that leptin $(10 \mu \mathrm{g} / \mathrm{ml})$ alone or in combination with IL-1 $\beta$ could induce cartilage destruction by activating NF-KB, ERK, JNK, and p38 in human articular chondrocytes, whose effect was inhibited by EPA and DHA. Fa-Bo Feng et al.[35] found that artesunate inhibited chondrocyte proliferation and accelerated cell apoptosis and autophagy via the suppression of the PI3K/AKT/mTOR signaling pathway.

Thus, this study showed that DHA could promote chondrocyte proliferation, decrease cell apoptosis, and increase autophagic activity in inflammatory articular chondrocytes. These effects of DHA could reduce the damage and degeneration of articular cartilage. This process was accomplished by inhibiting the expression of JNK and p38 signaling pathways. The effect of DHA could be blocked by autophagy inhibitor 3-MA. This study revealed that DHA could be used as a potential drug for the treatment of OA, 
which could alleviate the degradation of articular cartilage and delay the progression of OA. However, further studies are required to elucidate the intrinsic mechanisms involved in this process.

\section{Declarations}

\section{Funding}

This study was supported by the project of the Beijing Natural Science Foundation (No. 7192045).

\section{Competing Interests}

The authors have no relevant financial or non-financial interests to disclose.

\section{Author Contributions}

All authors contributed to the study conception and design, especially Lifeng Ma and Ai Guo. Material preparation, data collection were performed by Zishun Gong, Haomiao Yu, Guodong Wang and Ruiqi Cao. Data analysis was performed by Zishun Gong, Haomiao Yu and Heyong Yin. The first draft of the manuscript was written by Zishun Gong and Haomiao Yu, and all authors commented on previous versions of the manuscript. All authors read and approved the final manuscript. Zishun Gong and Haomiao Yu are the co-first authors of this paper.

\section{Ethics approval}

Ethics approval was granted by the Bioethics Committee of Beijing Friendship Hospital, Capital Medical University, China (approval code 2020-P2-205-01).

\section{Consent to participate}

Informed consent was obtained from all patients included in the study before surgery.

\section{Consent to publish}

No individual person's data was included in this research.

\section{References}

1. Bijlsma JW, Berenbaum F, Lafeber FP (2011) Osteoarthritis: an update with relevance for clinical practice. Lancet 377(9783):2115-2126

2. Murphy L, Helmick CG (2012) The impact of osteoarthritis in the United States: a population-health perspective: A population-based review of the fourth most common cause of hospitalization in U.S. adults. Orthop Nurs 31(2):85-91

3. Vincenti MP (2001) The matrix metalloproteinase (MMP) and tissue inhibitor of metalloproteinase (TIMP) genes. Transcriptional and posttranscriptional regulation, signal transduction, and cell-type- 
specific expression. Methods Mol Biol 151:121-148

4. Musumeci G, Castrogiovanni P, Trovato F et al (2015) Biomarkers of chondrocyte apoptosis and autophagy in osteoarthritis. Int J Mol Sci 16:20560-20575

5. Hwang HS, Hyun Ah Kim (2015) Chondrocyte Apoptosis in the Pathogenesis of Osteoarthritis. Int J Mol Sci 16(11):26035-26054

6. Caramés B, Olmer M, Kiosses WB, Lotz MK (2015) The relationship of autophagy defects to cartilage damage during joint aging in a mouse model. Arthritis Rheumatol 67(6):1568-1576

7. Thomas Riffelmacher FC, Richter, Anna Katharina Simon (2018) Autophagy dictates the metabolism and differentiation of inflammatory immune cells. Autophagy 14(2):199-206

8. Philip C Calder (2006) Polyunsaturated fatty acids and inflammation. Prostaglandins Leukot Essent Fatty Acids 75(3):197-202

9. De Caterina R, Cybulsky MI, Clinton SK, Gimbrone MA Jr, Libby P (1994) The omega-3 fatty acid docosahexaenoate reduces cytokine-induced expression of proatherogenic and proinflammatory proteins in human endothelial cells. Arterioscler Thromb 14(11):1829-1836

10. Calvo E, Palacios I, Delgado E, Sanchez-Pernaute O, Largo R, Egido J et al (2004) Histopathological correlation of cartilage swelling detected by magnetic resonance imaging in early experimental osteoarthritis. Osteoarthritis Cartilage 12:878-886

11. Tiraloche G, Girard C, Chouinard L, Sampalis J, Moquin L, Ionescu M et al (2005) Effect of oral glucosamine on cartilage degradation in a rabbit model of osteoarthritis. Arthritis Rheum 52:11181128

12. Loeser RF, Goldring SR, Scanzello CR et al (2012) Osteoarthritis: a disease of the joint as an organ. Arthritis Rheum 64(6):1697-1707

13. Mahajan A, Verma S, Tandon V (2005) Osteoarthritis. J Assoc Physicians India 53:634641

14. Blagojevic M, Jinks C, Jeffery A, Jordan KP (2010) Risk factors for onset of osteoarthritis of the knee in older adults: A systemic review and metaanalysis. Osteoarthritis Cartilage 18:2433

15. Loeser RF (2006) Molecular mechanisms of cartilage destruction: Mechanics, inflammatory mediators, and aging collide. Arthritis Rheum 54:13571360

16. Garver MJ, Focht BC, Taylor SJ (2015) Integrating lifestyle approaches into osteoarthritis care. J Multidiscip Healthc 8:409-418

17. Houard X, Goldring MB, Berenbaum F (2013) Homeostatic mechanisms in articular cartilage and role of inflammation in osteoarthritis. Curr Rheumatol Rep 15:375

18. Artemis PS (2002) Omega-3 fatty acids in inflammation and autoimmune diseases. J Am Coll Nutr 21(6):495-505

19. Calder PC (2010) Omega-3 fatty acids and inflammatory processes. Nutrients 2(3):355-374

20. Gabbs M, Leng S, Devassy JG, Monirujjaman M, Aukema HM (2015) Advances in our understanding of oxylipins derived from dietary PUFAs. Adv Nutr 6(5):513-540 
21. Pu H, Guo Y, Zhang W, Huang L, Wang G, Liou AK, Zhang J, Zhang P, Leak RK, Wang Y, Chen J, Yanqin Gao (2013) Omega-3 polyunsaturated fatty acid supplementation improves neurologic recovery and attenuates white matter injury after experimental traumatic brain injury. J Cereb Blood Flow Metab 33(9):1474-1484

22. Gorjão R, Azevedo-Martins AK, Rodrigues HG, Abdulkader F, Arcisio-Miranda M, Procopio J, Curi R (2009) Comparative effects of DHA and EPA on cell function. Pharmacol Ther 122(1):56-64

23. Renier G, Skamene E, DeSanctis J, Radzioch D (1993) Dietary n-3 polyunsaturated fatty acids prevent the development of atherosclerotic lesions in mice. Modulation of macrophage secretory activities. Arterioscler Thromb 13(10):1515-1524

24. Wang Zhenzhong G, Ai M, Lifeng Yu, Haomiao Z, Liang M, Hai C, Yinpeng Yu, Fei, Yang Bo (2016) Docosahexenoic acid treatment ameliorates cartilage degeneration via a p38 MAPK-dependent mechanism. Int J Mol Med 37(6):1542-1550

25. Mehler SJ, May LR, King C, Harris WS, Shah Z (2016) A prospective, randomized, double blind, placebo-controlled evaluation of the effects of eicosapentaenoic acid and docosahexaenoic acid on the clinical signs and erythrocyte membrane polyunsaturated fatty acid concentrations in dogs with osteoarthritis. Prostaglandins Leukot Essent Fatty Acids 109:1-7

26. Xu F, Song Y, Ai Guo (2019) Anti-Apoptotic Effects of Docosahexaenoic Acid in IL-1 $\beta$-Induced Human Chondrosarcoma Cell Death through Involvement of the MAPK Signaling Pathway. Cytogenet Genome Res 158(1):17-24

27. Joerg Gruenwald E, Petzold R, Busch H-P, Petzold H-J, Graubaum (2009) Effect of glucosamine sulfate with or without omega-3 fatty acids in patients with osteoarthritis. Adv Ther 26(9):858-871

28. Musumeci G, Loreto C, Carnazza ML, Martinez G (2011) Characterization of apoptosis in articular cartilage derived from the knee joints of patients with osteoarthritis. Knee Surg Sports Traumatol Arthrosc 19(2):307-313

29. Galanti C, Musumeci G, Valentino J, Giunta S, Castorina S (2013) A role for apoptosis in temporomandibular joint disc degeneration. A contemporary review. Ital J Anat Embryol 118(1):151158

30. Giuseppe Musumeci P, Castrogiovanni FM, Trovato AM, Weinberg, Mohammad K, Al-Wasiyah MH, Alqahtani A, Mobasheri (2015) Biomarkers of Chondrocyte Apoptosis and Autophagy in Osteoarthritis. Int J Mol Sci 16(9):20560-20575

31. ZhouPH LiuSQ, PengH (2008) The effect of hyaluronic acid on IL-1beta-induced chondrocyte apoptosis in a rat model of osteoarthritis. J Orthop Res Ther 26(12):1643-1648

32. HwangHS KimHA (2015) Chondrocyte apoptosis in the pathogenesis of osteoarthritis. Int J Mol Sci $16(11): 26035-26054$

33. WangSN XieGP et al (2015) Aucubin prevents interleukin-1beta induced inflammation and cartilage matrix degradation via inhibition of NF-kappaB signaling pathway in rat articular chondrocytes. Int Immunopharmacol 24(2):408-415 
34. Sabrina Ceccariglia A, Cargnoni AR, Silini O Parolini (2020) Autophagy: a potential key contributor to the therapeutic action of mesenchymal stem cells. Autophagy 16(1):28-37

35. Feng F-B, Hai-Yan Qiu (2018) Effects of Artesunate on chondrocyte proliferation, apoptosis and autophagy through the PI3K/AKT/mTOR signaling pathway in rat models with rheumatoid arthritis. Biomed Pharmacother 102:1209-1220

36. Deretic V, Levine B (2018) Autophagy balances inflammation in innate immunity Autophagy 14(2):243-251

37. Hwang HS, Kim HA (2015) Chondrocyte Apoptosis in the Pathogenesis of Osteoarthritis. Int J Mol Sci 16(11):26035-26054

38. Yeh P-S, Wang W, Chang Y-A, Lin C-J, Wang J-J, Chen R-M (2016) Honokiol induces autophagy of neuroblastoma cells through activating the $\mathrm{PI} 3 \mathrm{~K} / \mathrm{Akt} / \mathrm{mTOR}$ and endoplasmic reticular stress/ERK1/2signaling pathways and suppressing cell migration. Cancer Lett 370(1):66-77

39. Jeon H, Im GI (2017) Autophagy in osteoarthritis Connect Tissue Res 58(6):497-508

40. Kabeya Y, Mizushima N, Ueno T, Yamamoto A, Kirisako T, Noda T, Kominami E, Ohsumi Y, Yoshimori T (2000) LC3, a mammalian homologue of yeast Apg8p, is localized in autophagosome membranes after processing. EMBO J 19(21):5720-5728

41. Cheng NT, Meng H, Ma LF, Zhang L, Yu HM, Guo A (2017) Role of autophagy in the progression of osteoarthritis: The autophagy inhibitor, 3-methyladenine, aggravates the severity of experimental osteoarthritis. Int J Mol Med 39(5):1224-1232

42. Cheng NT, Guo A, Cui YP (2016) Intra-articular injection of Torin 1 reduces degeneration of articular cartilage in a rabbit osteoarthritis model. Bone Joint Res 5(6):218-224

43. Cheng NT, Guo A, Meng H (2016) The protective role of autophagy in experimental osteoarthritis, and the therapeutic effects of Torin 1 on osteoarthritis by activating autophagy. BMC Musculoskelet Disord 17:150

44. Wann AK, Mistry J, Blain EJ, Michael-Titus AT, Knight MM (2010) Eicosapentaenoic acid and docosahexaenoic acid reduce interleukin-1 $\beta$-mediated cartilage degradation. Arthritis Res Ther 12(6):R207. doi:

$10.1186 /$ ar

3183.Epub 2010 Nov 8

45. Phitak T, Boonmaleerat K, Pothacharoen P, Pruksakorn D, Kongtawelert P (2018) Leptin alone and in combination with interleukin-1-beta induced cartilage degradation potentially inhibited by EPA and DHA. Connect Tissue Res 59(4):316-331

\section{Figures}




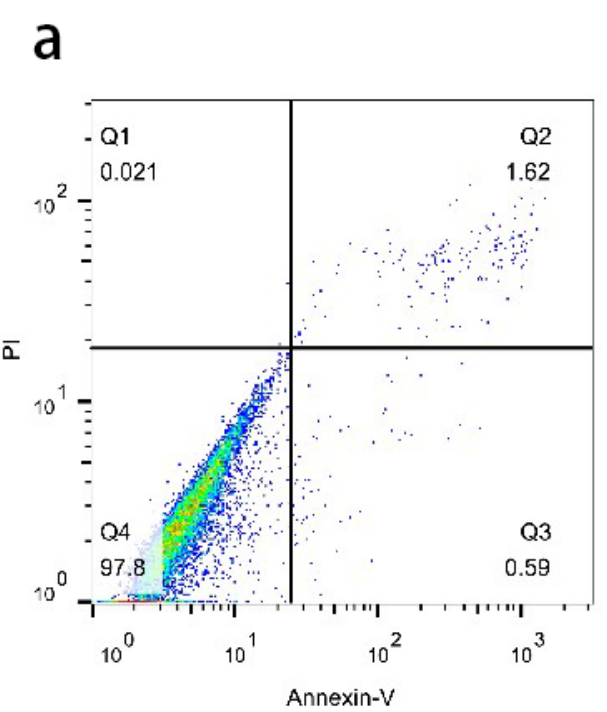

Control

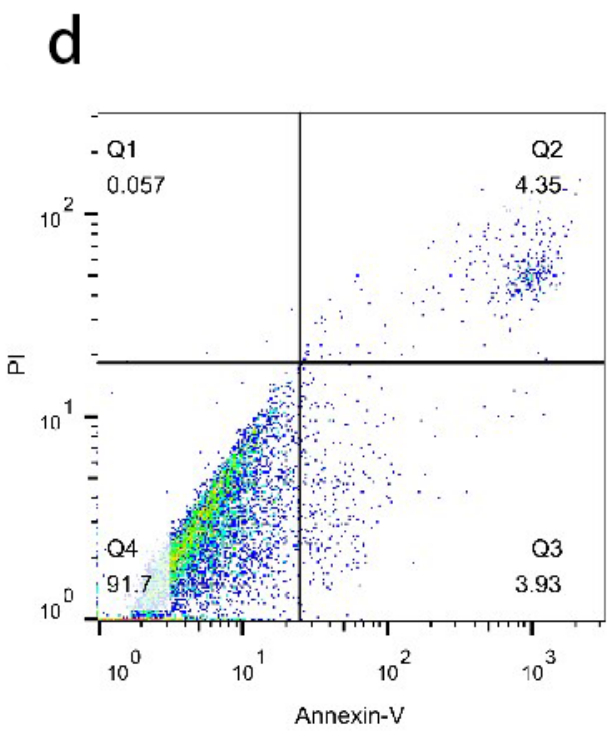

IL-1+ DHA+ 3-MA b

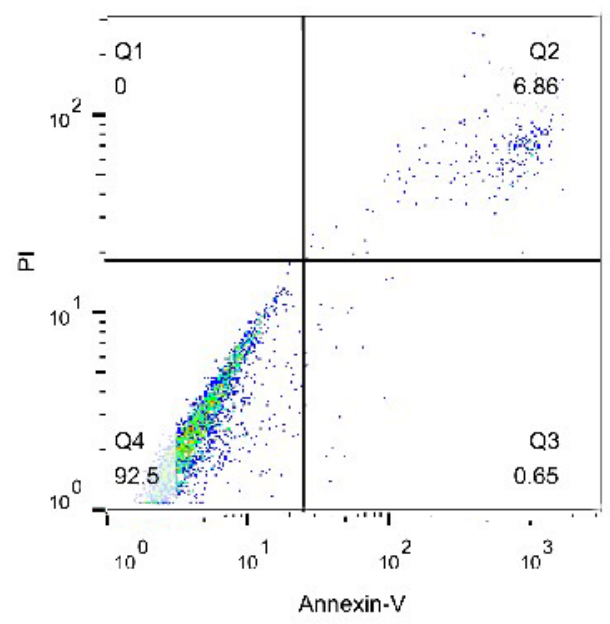

IL-1

e

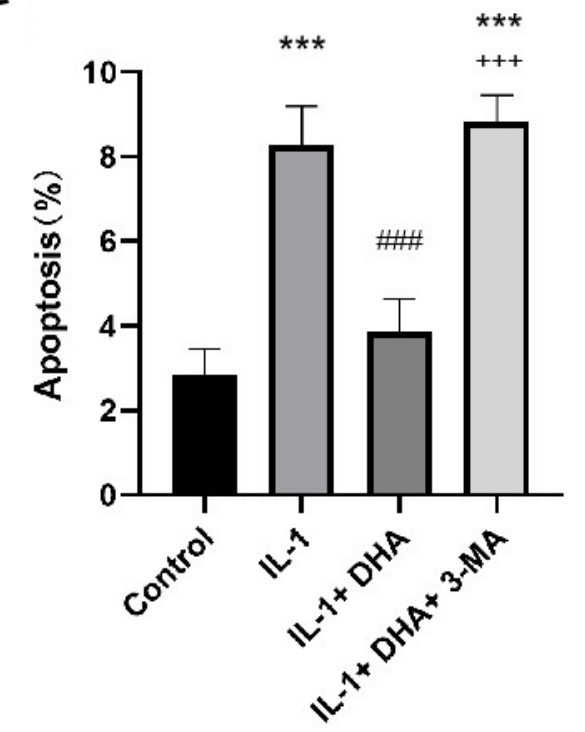

C

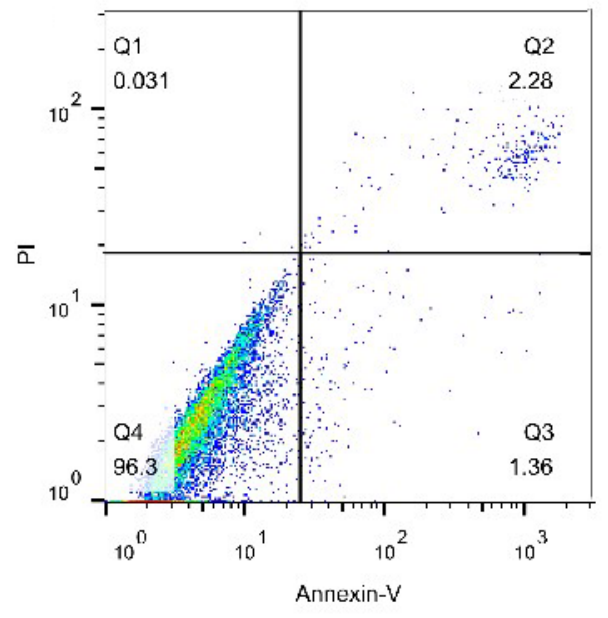

IL-1+ DHA
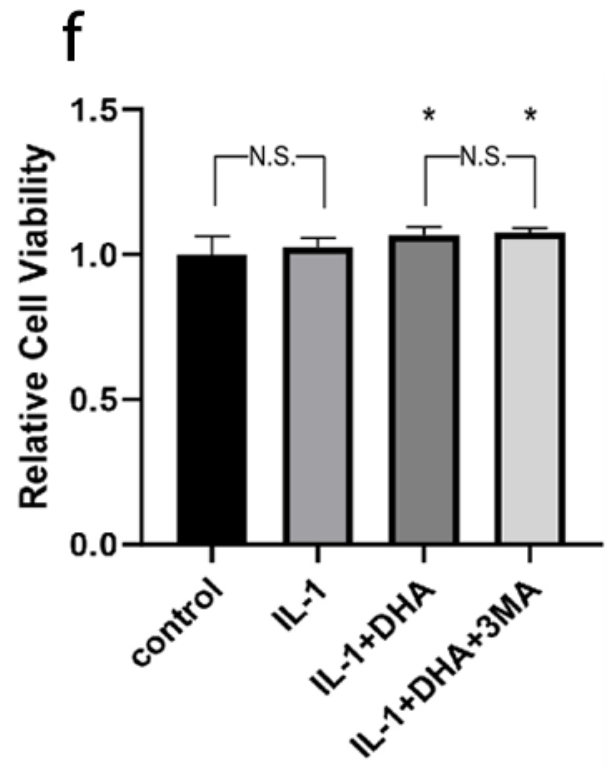

Figure 1

a-d. Flow cytometric detection of chondrocyte apoptosis between four groups $24 \mathrm{~h}$ after stimulation $(\mathrm{n}=$ 3). Apoptotic cells labeled with Annexin $\mathrm{V}$ and propidium iodide (PI) fluorescence were estimated. e. Apoptotic rates are shown by histogram. ${ }^{* \star} P<0.001$ compared to the control group, ${ }^{\# \#} P<0.001$ compared to the IL-1 group, ${ }^{+++} P<0.001$ compared to the IL-1 + DHA group. f. Comparison of the relative cell viability (RCV) in chondrocytes between four groups $24 \mathrm{~h}$ after stimulation $(n=5) .{ }^{*} P<0.05$ compared to the control group.

N.S., not significant. 


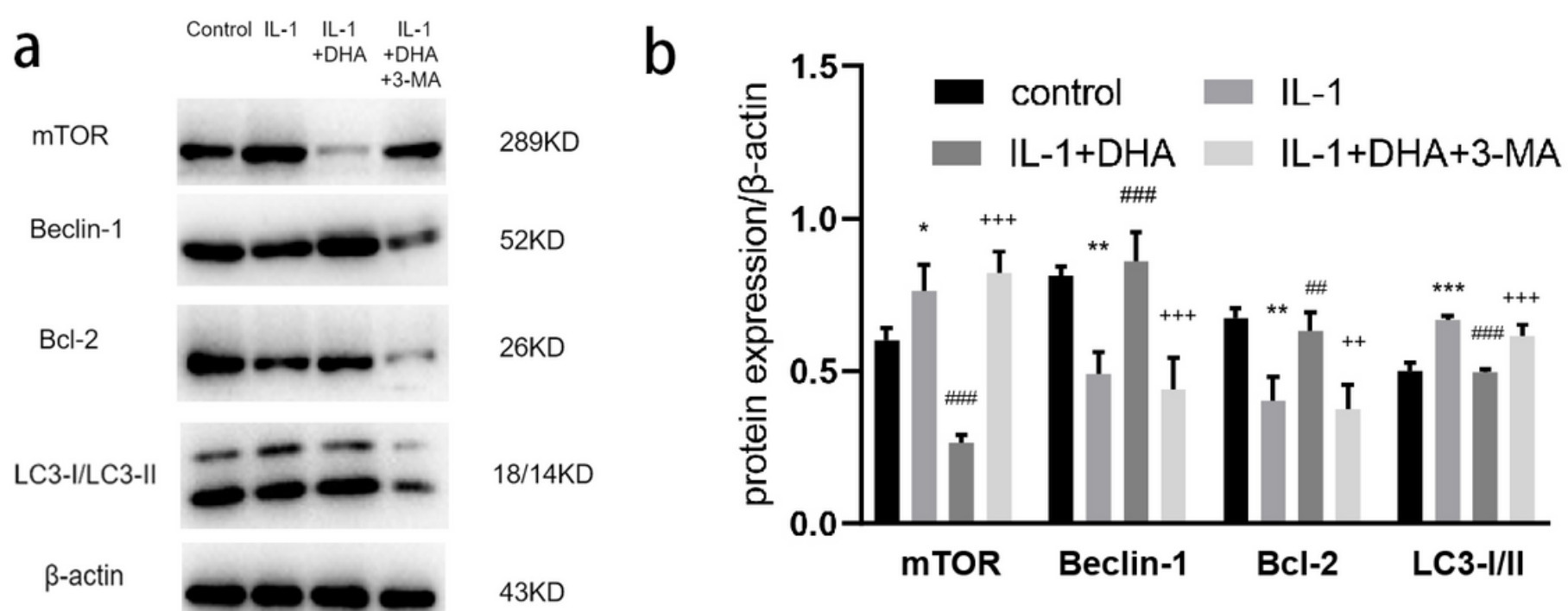

C

Control

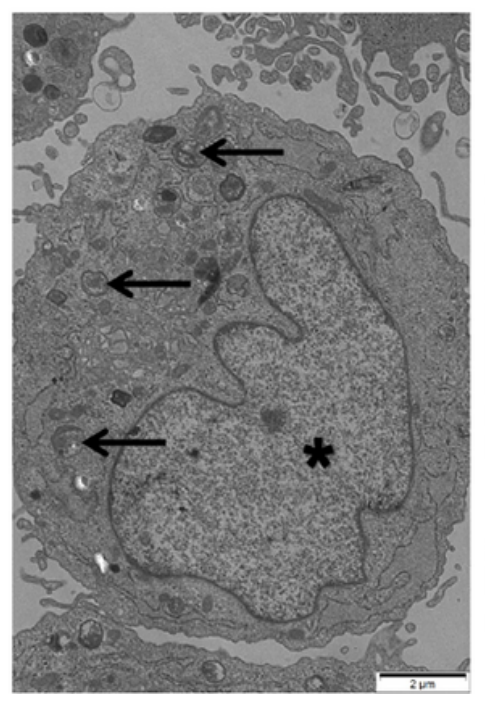

$\mathrm{IL}-1$

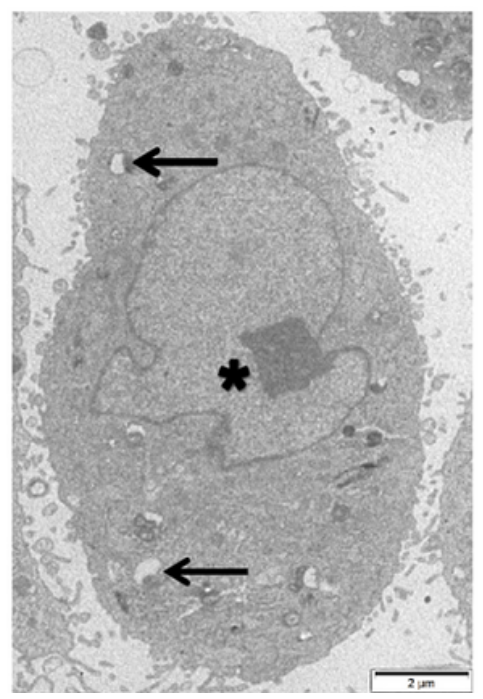

IL-1+DHA

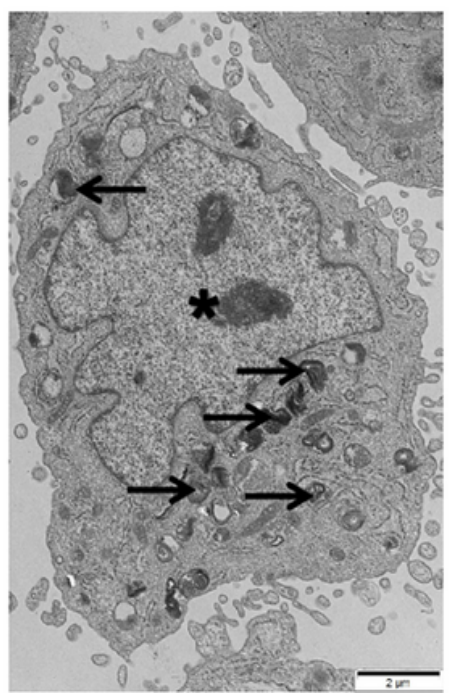

IL-1+DHA+3-MA

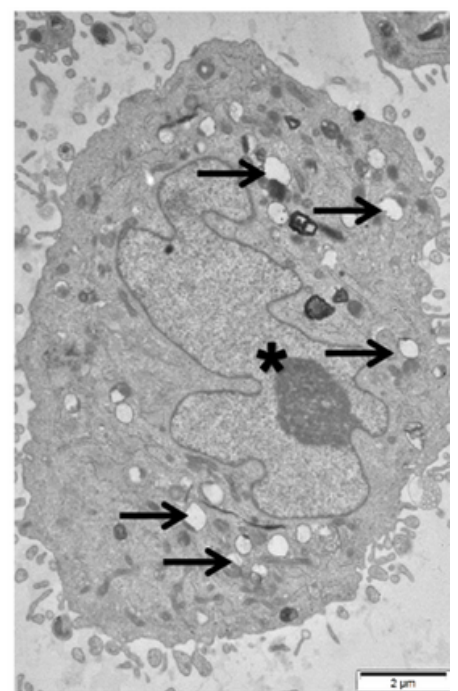

Figure 2

a.Expression of mTOR, Beclin-1, bcl2, and LC3-1/LC3-II in human chondrocytes. Control group: Normal

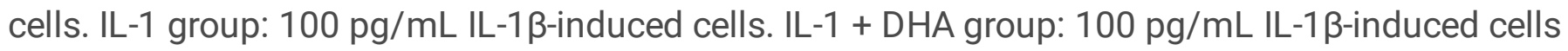
incubated with $50 \mathrm{mg} / \mathrm{mL}$ DHA. IL-1 + DHA + 3MA group: $100 \mathrm{pg} / \mathrm{mL}$ IL-1 $\beta$-induced cells incubated with $50 \mathrm{mg} / \mathrm{mL}$ DHA and $5 \mathrm{mmol} / \mathrm{L}$ 3-MA. Protein expression was evaluated by western blotting $(\mathrm{n}=3)$. b. Quantitative analysis shows that the mTOR expression and LC3-I/II ratio decreased while the expression of Beclin-1 and Bcl-2 increased in the IL-1 + DHA group compared to the IL-1 group. Conversely, mTOR expression and LC3-I/II ratio increased while Beclin-1 and Bcl-2 decreased in the IL-1 + DHA + 3MA group compared to the IL-1 + DHA group. ${ }^{\star} P<0.05{ }^{* \star} P<0.01,{ }^{* \star} P<0.001$ (compared to the control group), ${ }^{\#} P<$ $0.05,{ }^{\# \#} P<0.01,{ }^{\# \# \#} P<0.001$ (compared to the IL-1 group), ${ }^{+} P<0.05,{ }^{++} P<0.01,{ }^{+++} P<0.001$ (compared to the IL-1+DHA group). c. Representative images of transmission electron microscopy. Autophagic bodies were observed and marked with black arrows. The nuclei are marked with asterisks. Scale bar, 2 $\mu \mathrm{m}$. 

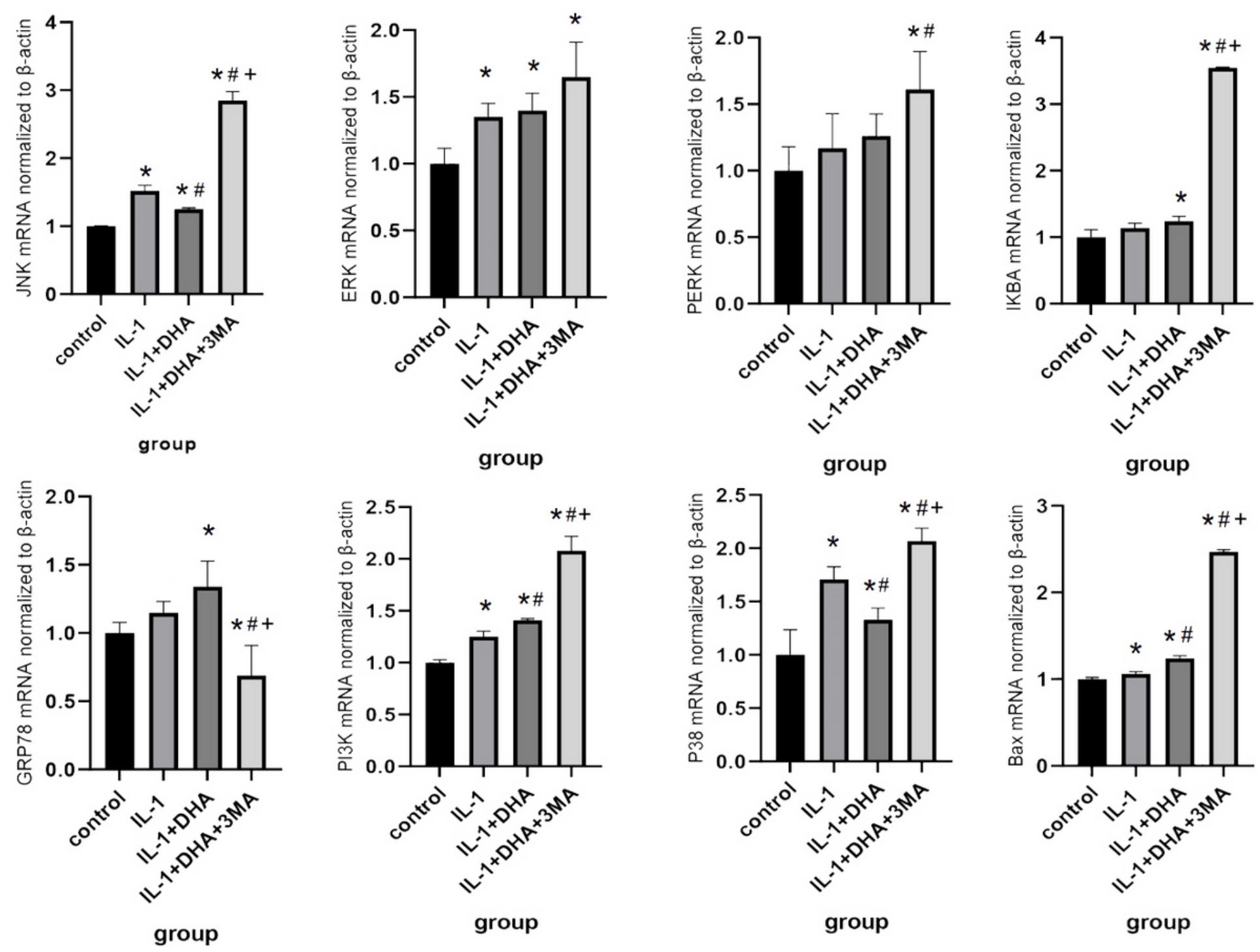

Figure 3

The mRNA expression of eight signaling pathway proteins (JNK, ERK, PERK, IKBA, GRP78, PI3K, p38, Bax) as detected by RT-PCR $(n=3)$. Compared to the control group, the mRNA expression of JNK, ERK, PI3K, p38, and Bax in the IL-1 group increased, while the addition of DHA reduced the expression of JNK and p38 compared to the IL-1 group. After intervention with autophagy inhibitor 3-MA, the expression of JNK and p38 increased significantly, suggesting that the effect of DHA was blocked by 3-MA. ${ }^{*} P<0.05$ (compared to the control group), ${ }^{\#} P<0.05$ (compared to the IL-1 group), ${ }^{+} P<0.05$ (compared to the IL$1+$ DHA group). 
a

Control

HE staining

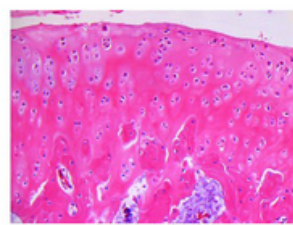

Toluidine blue

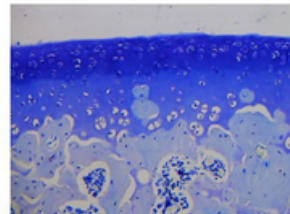

safranin-O FastGreen
OA
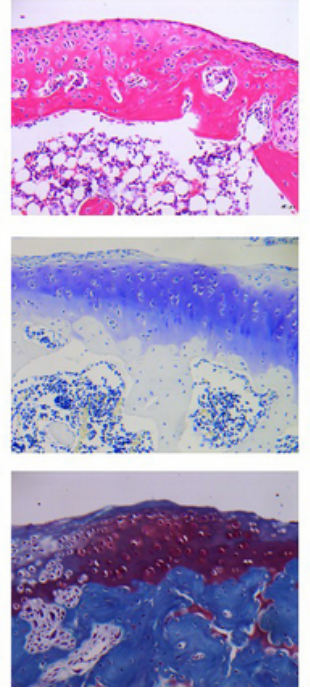

Sham + DHA
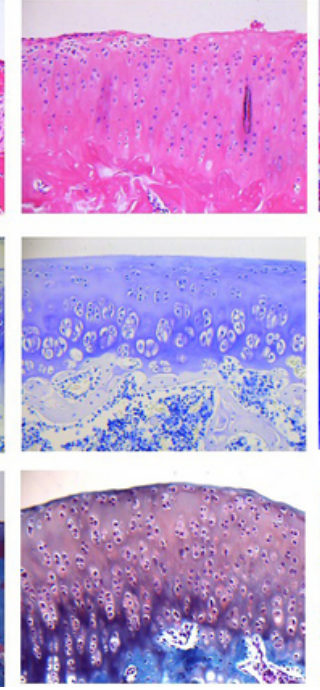

$\mathrm{OA}+\mathrm{DHA}$
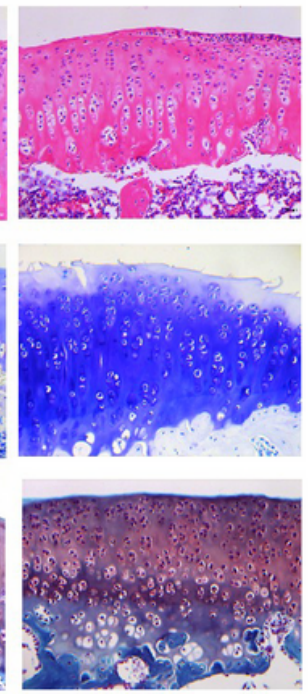

b

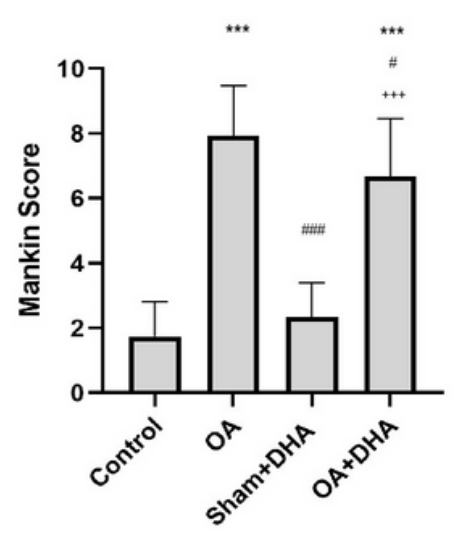

Figure 4

a. Representative sections of $\mathrm{H} \& \mathrm{E}$, toluidine blue, and safranin-O Fast Green staining of each group (original magnification 400x). b. Bar graph showing the results of modified Mankin score evaluation. The results are expressed as means \pm standard deviation (SD). ${ }^{\star \star \star} P<0.001$ compared to the control group, ${ }^{\#} P$ $<0.05,{ }^{\# \# \#} P<0.001$ compared to the IL-1 group, ${ }^{+++} P<0.001$ compared to the IL-1 + DHA group. 


\section{a Control}

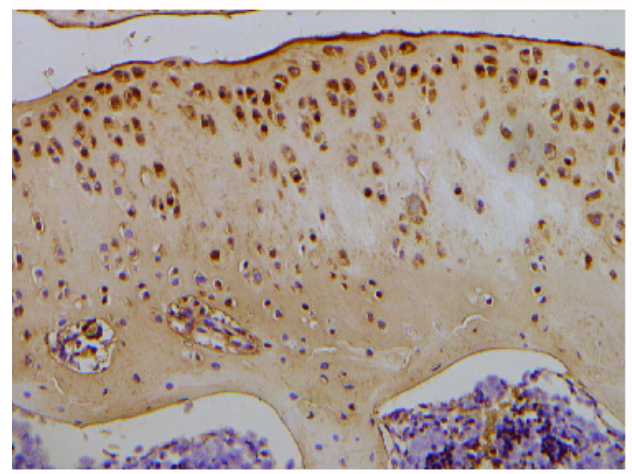

\section{$\mathrm{OA}+\mathrm{DHA}$}

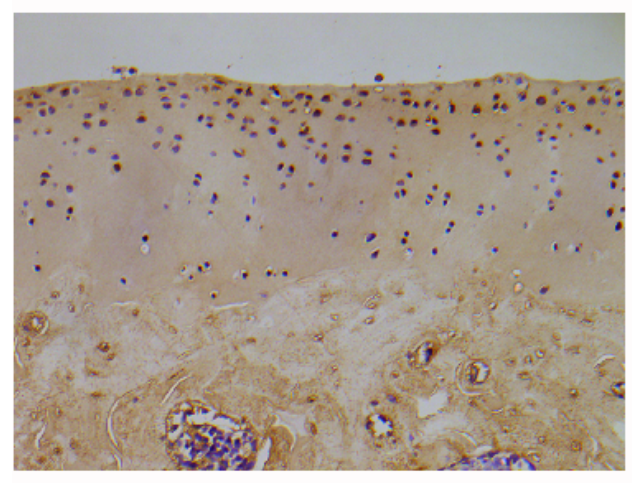

OA

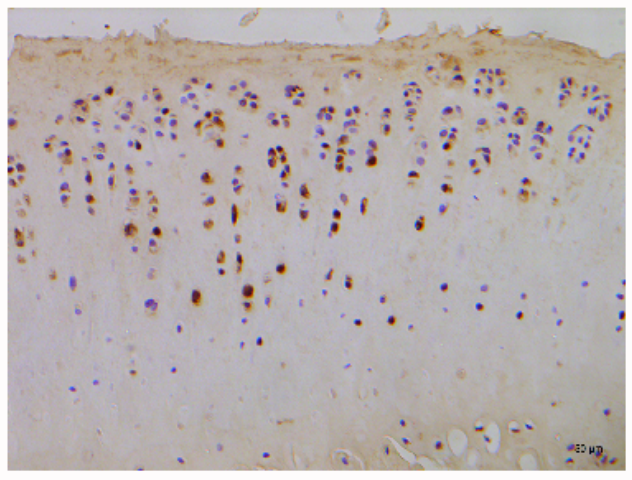

b

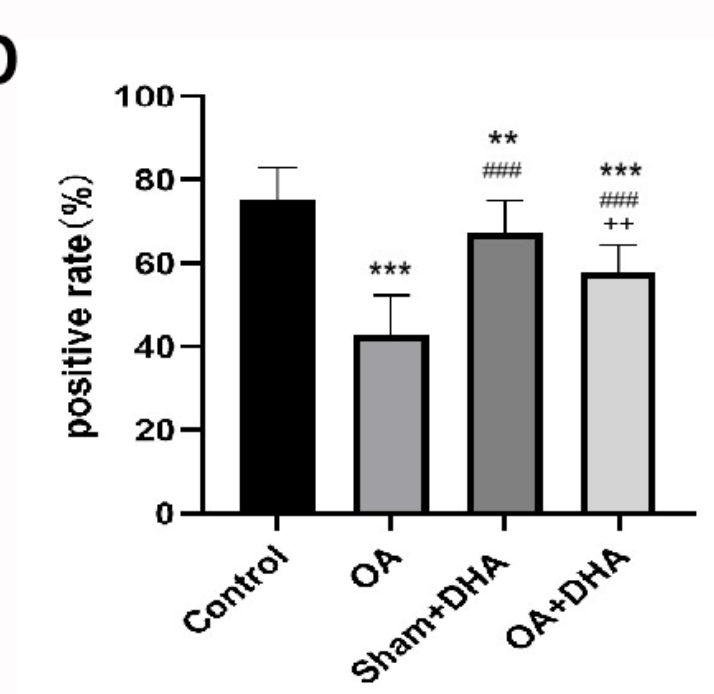

Sham + DHA

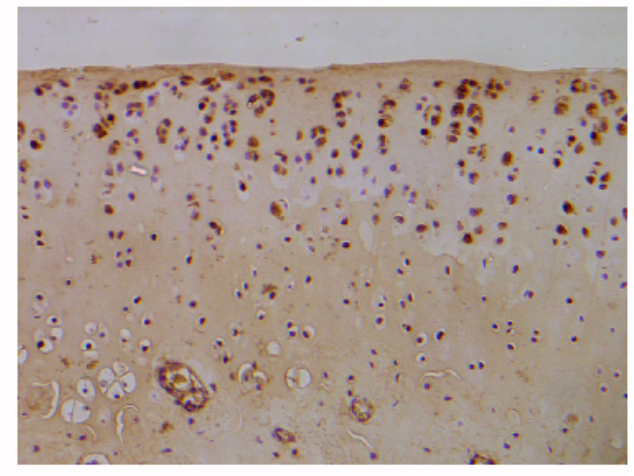

Figure 5

a. Representative sections of immunohistochemical analysis of collagen II in each group (original magnification 400x). b. Bar graph showing the results of positive rate. The results are expressed as mean \pm standard deviation (SD). ${ }^{* \star} P<0.01$, ${ }^{\star \star \star} P<0.001$ compared to the control group, ${ }^{\# \#} P<0.001$ compared to the IL-1 group, ${ }^{++} P<0.01$ compared to the IL-1 + DHA group. 